\title{
INCIDENCE OF MALIGNANCY IN INTESTINAL OBSTRUCTION AT OSMANIA GENERAL HOSPITAL
}

\section{Sri Divya Salkooti ${ }^{1}$, Praveen Mangalagiri *2.}

${ }^{1}$ Senior resident in General Surgery, Govt. Medical college, Nalgonda, Telangana, India.

${ }^{* 2}$ Assistant professor in General Surgery, Govt. Medical college, Nalgonda, Telangana, India.

\section{ABSTRACT}

Introduction: Intestinal obstruction (IO) is a common surgical emergency, which should be diagnosed urgently and promptly treated. The cause of $\mathrm{IO}$ has changed dramatically during the past century in developed countries. This will highlight the commonest causes of intestinal obstruction in the geographical location of the study which will suggest measures for prevention and treatment of the condition.

Aim and Objectives: The study was to determine the incidence of malignant bowel obstruction in patients presenting with intestinal obstruction, admitted in emergency department at a tertiary care center.

Materials and Methods: This study was conducted at Osmania General Hospital. It is a Prospective observational study that included 46 patients who were diagnosed to have acute intestinal obstruction features. The patients who are managed conservatively without surgical intervention are excluded.

Results: In the present study Malignant bowel obstruction ( $n=9)$ is the second most common cause of intestinal obstruction after postoperative adhesions $(n=10)$ at Osmania General Hospital. Malignant bowel obstruction is responsible for $19 \%$ of intestinal obstructions. 10 , overall has a poor prognosis if there is gangrene of bowel.

Conclusion: Patients presenting with $\mathrm{IO}$ due to malignancy have more morbidity and mortality and survival when compared to other causes. Early diagnosis of bowel malignancy by screening programmes and monitoring tumour markers will help in early diagnosis and prevent patients.

KEY WORDS: Adhesions, Intestinal obstruction, Malignant bowel and Stoma.

Address for correspondence: Dr. Praveen Mangalagiri, Assistant professor in General Surgery, Govt. Medical college, Nalgonda, Telangana, India. E-Mail: pmangalagiri@gmail.com

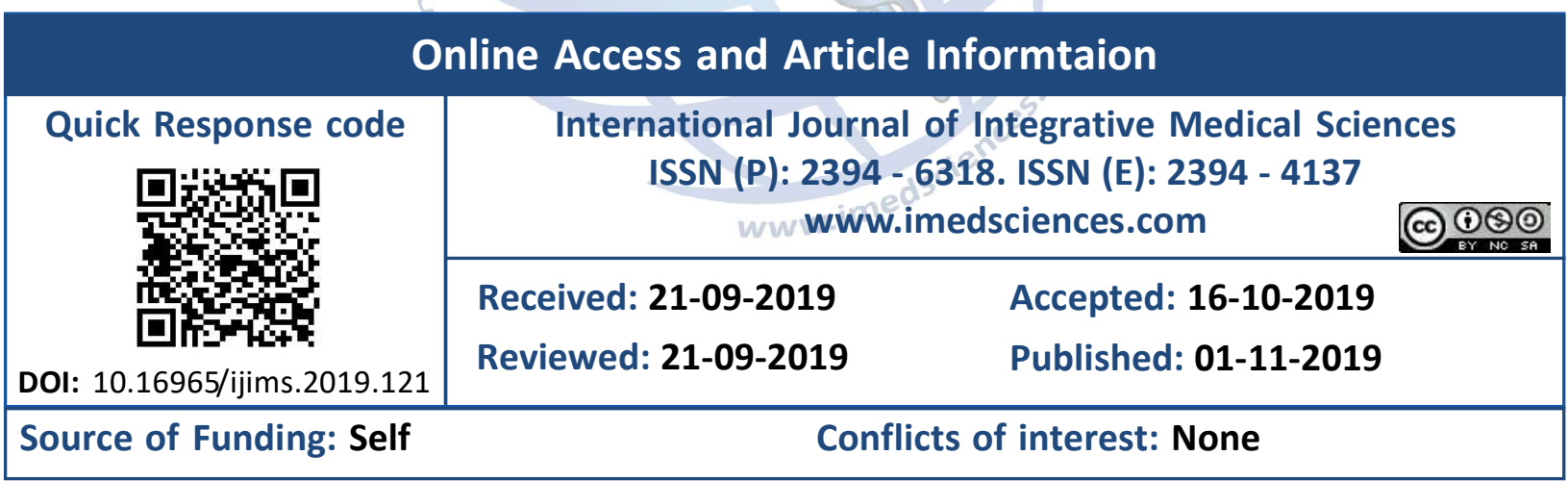

\section{INTRODUCTION}

One of the common acute abdominal emergencies is Intestinal obstruction (IO) i.e., $20 \%$ all admissions in surgical practice and in all age groups [1]. This is a major cause of morbidity among surgical cases all over the world. Early recognition and prompt intervention can prevent irreversible ischemia and thereby decrease the mortality and long-term morbidity $3 \%$ for simple obstructions to as great as $30 \%$ when there is vascular compromise or perforation of the obstructed bowel [2].

In western society past 50 years, there has been a considerable alteration in the pattern of 10 encountered, but the spectra reported varied widely [3]. While the main causes of IO may vary from country to country or among regions within a specific country, knowledge of the local disease patterns and outcomes may raise the index of suspicion, and reduce delays in diagnosis and intervention [4].

The post- operative adhesions and hernias cause extrinsic compression of the intestine are the most common causes, less common causes are tumors and strictures of the bowel which causes intrinsic blockage of the intestine for IO. 2 types 
of IO may be classified, dynamic and adynamic [5]. Adynamic obstruction is due to paralyzed bowel without any medical cause [5].

Despite multiple recent advances in diagnostic imaging and marked advances in our treatment armamentarium, IO will continue to occur [2]. Malignant tumors account for approximately $20 \%$ of the cases of small bowel obstruction [5]. The poor results associated with obstruction in malignancy may be as a consequence of operating upon frail old patients. This might be related to the pathological nature of their tumor, but also to the levels of skill brought to bear by the surgeon or to his choice of management in emergency. The etiology of intestinal obstruction varies widely across different areas in the world and also from one area to the other in our country itself $[3,4]$. I would like to study cause of intestinal obstruction in patients presenting to Osmania general hospital and determine the incidence of malignant bowel obstruction among them. In this study, we will discuss about the age, sex, incidence, etiology, clinical features, management, and outcomes of acute intestinal obstruction in adults.

\section{MATERIALS AND METHODS}

Osmania General Hospital being a tertiary care hospital caters to the needs of the patients from all over the states of Telangana, Andhra Pradesh and also from the adjacent states of Karnataka and Orissa. Such patient population provides ideal material for studying the disease at question in great detail. This Prospective observational study with 46 Patients (both the sex with age group 18-90years) presented to the emergency department with features of intestinal obstruction (Pain abdomen, Distention of abdomen, Vomiting, Radiological features of intestinal obstruction) to the acute surgical care during the study period (August 2016 to November 2017) and are managed by operative treatment were studied. Patients presenting with hollow viscus perforation, gastric outlet obstruction, nonoperative management symptoms were excluded from the study.

The mode of presentation, duration of complaints and status of patient at presentation were noted and the operative findings of the patients were taken as a basis for diagnosing abdomen, adhesiolysis with or without bowel resection was done. In cases with large bowel obstruction due to malignancy or volvulus, a proximal colostomy with or without resection of the bowel was done. In cases with small bowel malignancy, resection and anastomosis was done. Postoperative recovery and complications that occurred during the stay were also noted. Patients with complications spend more time in hospital than those whose recovery was uneventful. Therefore, postoperative hospital stays and complications were studied in the patients admitted with intestinal obstruction and the results are compared with already conducted studies to reach to conclusions. As stoma represent a major cause of distress to the patient and also require experts in stoma care for follow up, the number of patients requiring ileostomy or colostomy was also assessed in the present study.

\section{RESULTS}

Both the sex (male 36 and female 10) with age group 18-90 years patients included in the study majority of patients (19 patients of 46 ) belonged to the age group of 50-59, average age of the patients is 48 years. Most common cause of 10 was postoperative adhesions $(n=10)$ followed by obstructed inguinal hernia $(n=8)$ and large bowel malignancy in that order. Malignant bowel obstruction $(n=9)$ is the second most common cause of intestinal obstruction in the present study if both small bowel and large bowel malignancies are considered together.

Fig. 1: Showing the common cause of Intestinal Obstruction.

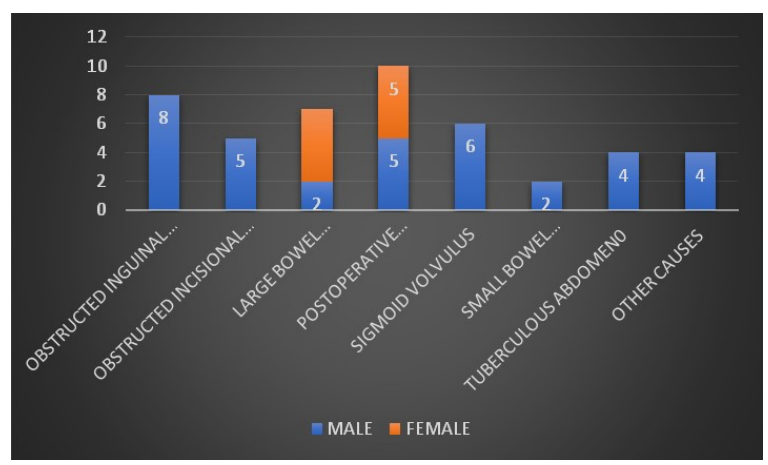

Table 1: Clinical Course of Patients with Malignancy in the Present Study.

\begin{tabular}{|l|c|c|c|}
\hline & $\begin{array}{c}\text { Postoperative } \\
\text { Hospital Stay (days) }\end{array}$ & Complications & Death \\
\hline Small bowel tumours & 13 & NO & 0 \\
\hline Carcinoma descending colon & 12 & 2 & 1 \\
\hline Carcinoma sigmoid colon & 14 & NO & 0 \\
\hline Carcinoma rectum & 24 & 1 & 1 \\
\hline
\end{tabular}


Fig. 2: Showing Average Duration of Hospital Stay.

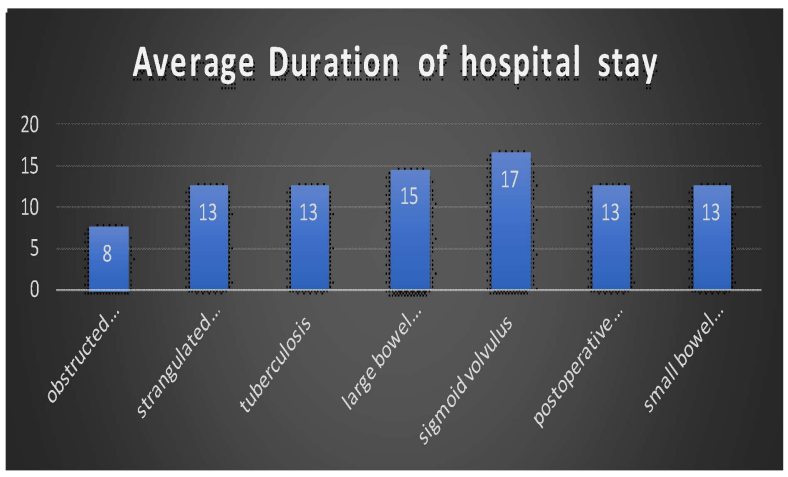

The average duration of hospital stay was 13 days, Patients admitted with sigmoid volvulus and large bowel malignancy had an average duration of hospital stay of 17 and 15 days respectively, Postoperative complications like wound infection and wound dehiscence were responsible for the prolonged duration of admission, Patients admitted with obstructed inguinal hernia had the shortest average duration of stay at the hospital. Also, patients admitted with arterial and venous thrombosis diseases had a prolonged hospital stay of up to 30 days. this accounts for the longest hospital stay.

Fig. 3: Showing proportion of patients requiring stoma

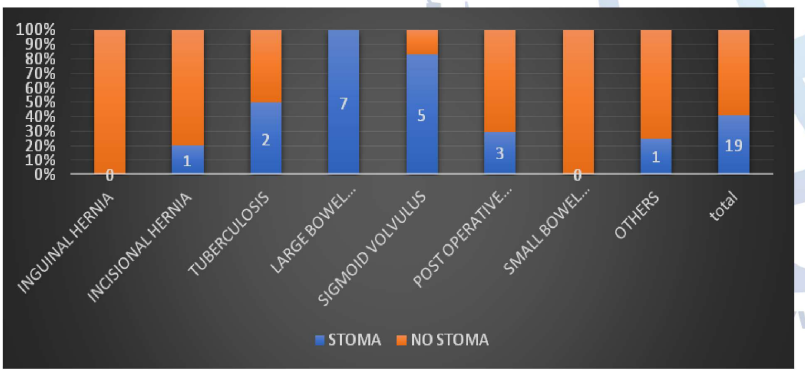

19 patients of 46 admitted with intestinal obstruction required creation of stoma in view of distal unresectable tumour or peritoneal contamination due to bowel gangrene or unhealthy, unsuitable bowel for distal anastomosis. The maximum number of stomas(i.e.) 7 were created in view of large bowel malignancy followed by sigmoid volvulus.
Fig. 4: Showing proportion of patients with bowel gangrene.

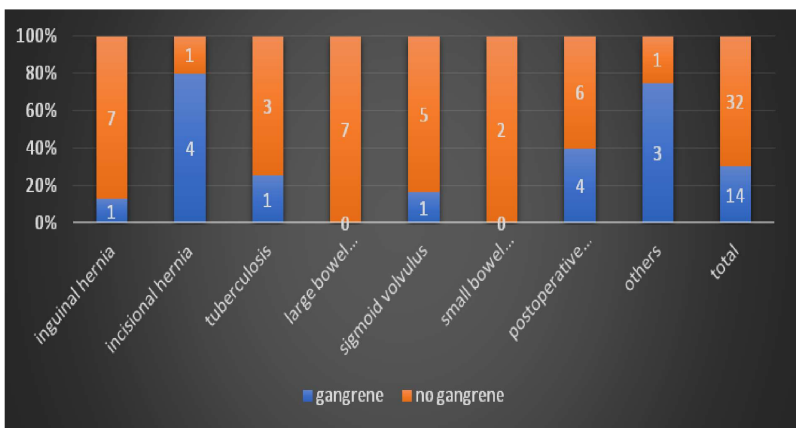

14 out of 46 patients presenting with obstruction had intraoperative finding of gangrene bowel was noted. Gangrene of bowel occurred more often due to strangulated incisional hernia and postoperative adhesions causing bowel obstruction each of which causes was responsible for 4 cases respectively. The gangrenous part was resected and either a stoma was created or the continuity restored by primary anastomosis.

Fig. 5: Showing postoperative morbidity and mortality.

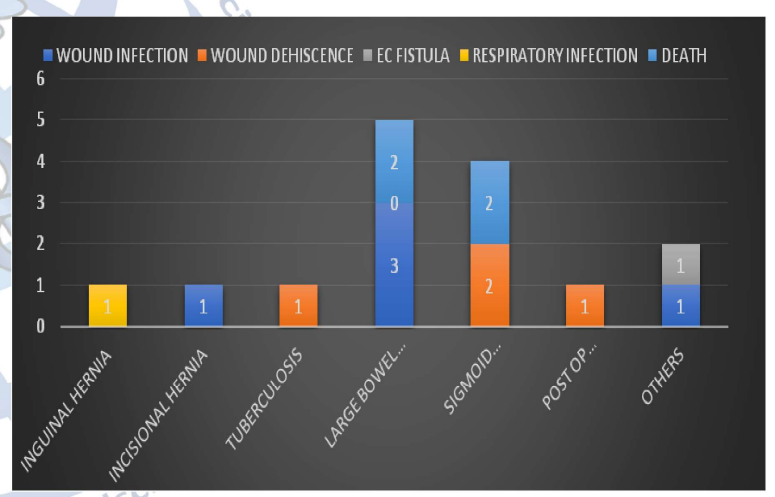

The most common complication during the postoperative period was wound infection followed by wound dehiscence. And complications occurred in patients with stoma more often than the patients without a stoma. A total of 4 patients out of the 46 chosen for the study died during the postoperative period. Two patients with carcinoma rectum and two with sigmoid volvulus died in the postoperative period.

\begin{tabular}{|c|c|c|c|c|c|c|c|}
\hline \multirow{9}{*}{$\begin{array}{l}\text { Table 2: Showing the } \\
\text { postoperative } \\
\text { complications. }\end{array}$} & & $\begin{array}{c}\text { Wound } \\
\text { infection }\end{array}$ & $\begin{array}{c}\text { Wound } \\
\text { dehiscence }\end{array}$ & $\begin{array}{c}\text { Enterocutaneous } \\
\text { fistula }\end{array}$ & Death & Others & Total \\
\hline & Obstructed inguinal hernia & 0 & 0 & 0 & 0 & 1 & 1 \\
\hline & Strangulated incisional hernia & 0 & 0 & 0 & 0 & 0 & 0 \\
\hline & Tuberculosis & 0 & 1 & 0 & 0 & 0 & 1 \\
\hline & Large bowel malignancy & 3 & 0 & 0 & 2 & 0 & 5 \\
\hline & Sigmoid volvulus & 0 & 2 & 0 & 2 & 0 & 4 \\
\hline & Post-operative adhesions & 0 & 1 & 0 & 0 & 0 & 1 \\
\hline & Small bowel malignancy & 0 & 0 & 0 & 0 & 0 & \\
\hline & Other causes & 1 & 0 & 1 & 0 & 0 & 2 \\
\hline
\end{tabular}




\section{DISCUSSION}

ntestinal obstruction is one of the commonly encountered clinical entities. The mortality has reduced significantly and $1-4 \%$ of mortality in emergency surgeries is contributed by acute intestinal obstruction. The following were the observations made from the study of 46 patients of acute intestinal obstruction in adults at Osmania General Hospital, Hyderabad, Telangana. $\mathrm{IO}$ is a common surgical emergency encountered in the surgical practice, and many studies have been published regarding the etiology and pathophysiology. But there is a changing trend of intestinal obstruction with an increase in the adhesive obstruction and a concomitant decrease in the incidence of obstructed hernias has been observed by Mohamed A et al [4] in a study carried out in India.

Incidence: The most common cause of 10 in the present study is postoperative adhesions $21.7 \%$ of cases followed by $19 \%$ malignant tumours causing of obstruction cases, obstructed inguinal hernia causing $17.3 \%$ of cases and tuberculosis in $8 \%$ of cases. The most common cause of intestinal obstruction in our study is postoperative adhesions, that is in concordance with Asad S et al [6] and Markogiannakis $\mathrm{H}$ et al [7]. This reflects the changing global trends in etiology if intestinal obstruction with increasing trend of postoperative adhesions as the leading cause of intestinal obstruction. ${ }^{8}$ The incidence of malignancy in intestinal obstruction varied widely in the studies examined from $5.6 \%$ in Asad S et $\mathrm{al}^{6}$ to $13 \%$ in Markogiannakis $\mathrm{H}$ et al [7]. The incidence of malignancy according to our study is higher than the other studies from elsewhere, which might be an error due to the small sample size or is truly reflecting the higher incidence of malignant bowel obstruction in our area which needs further scrutiny for substantiation. Colorectal cancer is the third most common cancer in men and the second in women globally [8]. There is also an increase in incidence of malignant bowel obstruction, as reflected by our study. The most common cause of intestinal obstruction in Phillipo et al [9] study is obstructed hernia, which could be because of either a greater prevalence of intestinal obstruction in that part of the world or due to lower frequency of surgical treatment of uncomplicated hernias, leading to obstruction and other complications. Preventive measures for reducing the burden of colorectal cancer in both low- and high-resource countries include being physically active, maintaining a healthy body weight, minimizing consumption of red meat and alcohol, maximizing consumption of fruit and vegetables, and cessation of smoking. Highresource countries could benefit from the implementation of colorectal screening that allows detection and removal of precancerous polyps and early-stage cancers. However, few countries (Czech Republic, Germany, Israel, Japan, and Poland) have national colorectal cancer screening programs; most have screening initiatives consisting of recommendations and/ or guidelines with opportunistic screening or pilot studies [10].

The variable and slow growth of adenomatous polyps and colorectal cancers coupled to the previously mentioned incidence rates of colorectal cancer merit aggressive screening approaches. Debate is vigorous as to the best approaches, and multiple factors influence that decision: (i) simplicity and rapidity so as to enhance patient compliance, (ii) benefit-risk ratio, (iii) sensitivity, (iv) specificity, and (v) costeffectiveness and other economic factors [11].

Currently to that end, optical colonoscopy likely offers the most effective approach when one considers all of these factors. The average-risk patient is defined as male or female above the age of 50 without personal or family history of adenomatous polyps or colorectal cancer, and also absence of any occult or acute GI bleeding. Historically, several screening modalities have been advocated, and these include the following: digital rectal examination, fecal occult blood testing, sigmoidoscopy (historically, this was rigid and has been supplanted by flexible sigmoidoscopy), barium enema, and colonoscopy. Optical colonoscopy is currently the most sensitive method for screening [12]. Advantages include direct visualization, ability to remove polyps with rate-limiting factors of size and anatomic location, and obtaining biopsies. Disadvantages involve the preparation, invasive nature of the procedure, and potential side effects that include perforation [13]. 
The digital rectal examination should be part of the general physical examination. Anorectal masses may be palpated. Flexible sigmoidoscopy does not require conscious sedation and hemodynamic monitoring, and will typically allow visualization of the rectum, sigmoid colon, and descending colon to the splenic flexure. Flexible sigmoidoscopy should not be considered as a single screening measure but requires coupling with barium enema. Barium enema permits visualization of the entire colon and experience is necessary to ensure proper visualization of the rectum. Barium enema affords advantages of ease of preparation, lack of conscious sedation and hemodynamic monitoring, and ability to visualize polyps and masses. However, small polyps may be missed. Furthermore, if a luminal polyp or mass is identified, then colonoscopy will be necessary for polypectomy and/or biopsies. New noninvasive technologies are investigational but receiving attention in clinical studies, and these may provide some initial data demonstrating efficacy. These relate to CT-colonography (referred to as virtual colonoscopy). and even magnetic resonance-colonography. A recent study is consistent with the feasibility of virtual colonoscopy when compared with optical colonoscopy, although large-scale population studies are still needed [14].

Gangrene of bowel: In the present study 30\% of patients operated for intestinal obstruction had gangrenous bowel found intraoperatively, for which resection and primary anastomosis or stoma needed to be performed. Fevang BT et al ${ }^{15}$ was found $16 \%$, which might be due to the constricting effect of the neck of the hernia sac compromising the blood supply.

Duration of hospitalization: The highest mean duration of hospitalization was for patients admitted with large bowel obstruction with 17 days for sigmoid volvulus and 15 days for large bowel malignancy. The overall mean duration of hospitalization in the present study was 13 days. Chalya PL et al [9] study was found 26 days, Markogiannakis $\mathrm{H}$ et al [7] study was 7 days. The duration of hospital stay reflects the morbidity due to postoperative complications, with moribund patients requiring longer duration of hospital stay, and early discharge in patients with uneventful recovery.

Postoperative morbidity: In the present study, the rate of postoperative complications was $23 \%$ with postoperative wound infection and wound dehiscence as the two major complications. Other complications included enterocutaneous fistula and iatrogenic bladder injury, which occurred in one patient each. Fevang BT et al [15], Priscilla SB et al [5] study, and Asad S et al [6] were showed similar complication rate with postoperative wound infection as the most common complication to the present study. The patients with large bowel malignancy and large intestinal obstruction and large bowel obstruction had a greater complication rate while the patients presenting with obstructed inguinal hernia had uneventful postoperative period. This could be because of the malnourishment and low immunity of the patients with intestinal obstruction or due to the late presentation to the hospital.

Postoperative mortality: $8 \%$ of patients who underwent operation for intestinal obstruction died in the postoperative period due to severe complications like septic shock. Two of the seven patients diagnosed with large bowel malignancy died in postoperative period making up to $28 \%$ mortality. The mortality in the study conducted by Priscilla SB et al [5] was 7.8\%, which is closer to the present study.

\section{CONCLUSION}

The syndrome of 10 generates a large number of hospital visits and may affect the very young, the very old, either sex and all socioeconomic groups. The cause of 10 has changed dramatically during the past century in developed countries. In the present study Malignant bowel obstruction $(n=9)$ is the second most common cause of intestinal obstruction after postoperative adhesions $(n=10)$ at our institute. Malignant bowel obstruction is responsible for $19 \%$ of intestinal obstructions. Patients presenting with intestinal obstruction due to malignancy have more morbidity and mortality and survival when compared to other causes. Early diagnosis of bowel malignancy by screening programmes and monitoring tumour markers will help in early diagnosis and prevent patients from presenting with malignant bowel obstruction and suffer from its associated complications. 


\section{REFERENCES}

[1]. Tesfamichael G. Mariam, Addisu Taye Abate, Mehammed Adem Getnet. Surgical Management Outcome of Intestinal Obstruction and Its Associated Factors at University of Gondar Comprehensive Specialized Hospital, Northwest Ethiopia, 2018. Surgery Research and Practice. 2019:8.

[2]. Guido M Sclabas, George Asarosi; Small bowel obstruction; Maigots Abdominal Operation chapter 29, P.588.

[3]. McEntee G, Pender D, Mulvin D, McCullough M, Naeeder S, Farah S, et al. Current spectrum of intestinal obstruction. Br J Surg. 1987;74(11):97680.

[4]. Mohamed A, Sahoo N, Das SK, et al. Profile of operated acute intestinal obstruction patients at a tertiary health care institution. J. Evolution Med. Dent. Sci. 2017;6(15):1215-9

[5]. Priscilla SB, Edwin IA, Kumar K, Gobinath M, Arvindraj VM, Anandan H. A Clinical Study on Acute Intestinal Obstruction. Int J Sci Stud 2017;5(2): 107-10.

[6]. Asad S, Khan H, Khan IA, Ali S, Ghaffar S, Zia ur Rehman. Aetiological factors in mechanical intestinal obstruction. J Ayub Med Coll Abbottabad. 2011;23(3):26-7.

[7]. Markogiannakis $H$, Messaris E, Dardamanis D, Pararas N, Tzertzemelis D, Giannopoulos P, et.al. Acute mechanical bowel obstruction: clinical presentation, etiology, management and outcome. World J Gastroenterol. 2007;13(3):432-7.

[8]. Fausto Catena, Salomone Di Saverio, Federico Coccolini, Luca Ansaloni, Belinda De Simone, et.al. Adhesive small bowel adhesions obstruction: Evolutions in diagnosis, management and prevention. World J Gastrointest Surg. 2016; 8(3): 222-31.
[9]. Chalya PL, Mabula JB, Chandika AB, Giiti G. Dynamic bowel obstruction: aetiology, clinical presentation, management and outcome at Bugando Medical Centre, Mwanza, Tanzania. Tanzan J Health Res. 2014; 16(1):38-46.

[10]. Mercedes Navarro, Andrea Nicolas, Angel Ferrandez, Angel Lanas. Colorectal cancer population screening programs worldwide in 2016: An update.World J Gastroenterol. 2017; 23(20): 3632-42.

[11]. Jing Jin, 1 Grant Edward Sklar, Vernon Min Sen Oh, Shu Chuen Li. Factors affecting therapeutic compliance: A review from the patient's perspective. Ther Clin Risk Manag. 2008; 4(1): 269-286.

[12].Saleh A. Al-Enezi, Saqer A. Alsurayei, Ali E. Ismail, Nasser Yehia A. Aly, Waleed A. Ismail, Amany A. Abou-Bakr. Adenomatous Colorectal Polyps in Patients Referred for Colonoscopy in a Regional Hospital in Kuwait. Saudi J Gastroenterol. 2010 Jul; 16(3): 188-193.

[13]. P.H. Gordon. Screening for colorectal carcinoma. Curr Oncol. 2010 Apr; 17(2): 34-39.

[14]. Cary B Aarons, Skandan Shanmugan, Joshua IS Bleier. Management of malignant colon polyps: Current status and controversies. World J Gastroenterol. 2014; 20(43): 16178-83.

[15. Fevang BT, Fevang J, Stangeland L, Soreide O, Svanes $\mathrm{K}$, Viste A. Complications and death after surgical treatment of small bowel obstruction: A 35-year institutional experience. Ann Surg. 2000 Apr;231(4):529-37.

How to cite this article: Sri Divya Salkooti, Praveen Mangalagiri. INCIDENCE OF MALIGNANCY IN INTESTINAL OBSTRUCTION AT OSMANIA GENERAL HOSPITAL. Int J Intg Med Sci 2019;6(7):854-859. DOI: $10.16965 /$ ijims.2019.121 